\title{
(+)-Z-Bisdehydrodoisynolic Acid Enhances Basal Metabolism and Fatty Acid Oxidation in Female Obese Zucker Rats
}

\author{
William J. Banz, ${ }^{1,2}$ April D. Strader, ${ }^{2}$ Kolapo M. Ajuwon, ${ }^{3}$ Yuqing Hou, ${ }^{4}$ \\ Cal Y. Meyers, ${ }^{4}$ and Jeremy E. Davis ${ }^{1}$ \\ ${ }^{1}$ Department of Animal Science, Food and Nutrition, Southern Illinois University, Carbondale, IL 62901-4317, USA \\ ${ }^{2}$ Physiology, Southern Illinois University, Carbondale, IL 62901-6512, USA \\ ${ }^{3}$ Department of Animal Sciences, Purdue University, West Lafayette, IN 47907-2054, USA \\ ${ }^{4}$ Department of Chemistry and Biochemistry, Meyers Institute for Interdisciplinary Research in Organic and Medicinal Chemistry, \\ Southern Illinois University, Carbondale, IL 62901-4409, USA \\ Correspondence should be addressed to William J. Banz, banz@siu.edu
}

Received 18 July 2011; Revised 21 December 2011; Accepted 21 December 2011

Academic Editor: Jack A. Yanovski

Copyright (C) 2012 William J. Banz et al. This is an open access article distributed under the Creative Commons Attribution License, which permits unrestricted use, distribution, and reproduction in any medium, provided the original work is properly cited.

\begin{abstract}
We have previously reported that the synthetic estrogen, (+)-Z-bisdehydrodoisynolic Acid [(+)-Z-BDDA], attenuated weight gain and cardiovascular risk in obese rodents. To determine if these antiobesity effects were attributed to changes in basal metabolism, we assessed indirect calorimetry and metabolic profile in female obese Zucker (OZR) rats provided (+)-Z-BDDA (0.0002\% food admixture) for 11 weeks. Similar to our previous findings, (+)-Z-BDDA reduced weight gain and improved lipid and glucose homeostasis in OZR rats. Furthermore, resting energy expenditure was increased by $(+)-Z$-BDDA, as evident by heat production and oxygen consumption. We also observed a marked reduction in respiratory quotient (RQ) along with a corresponding induction of hepatic AMPK in rodents provided (+)-Z-BDDA. Collectively, these findings indicate that (+)-Z-BDDA partially attenuated obesity and associated pathologies through increased resting energy expenditure and fatty acid utilization. Further investigation is required to fully elucidate the mechanisms involved as well as to determine the potential therapeutic implications for (+)-Z-BDDA on obesity and its related pathologies.
\end{abstract}

\section{Introduction}

Doisynolic acids (DAs), such as (+)-Z-BDDA, are a group of synthetically produced compounds that exhibit distinct selective estrogen receptor modulator (SERM) activity in vivo [1]. Based on the structural similarity to $17 \beta$-estradiol, DAs were originally investigated as an alternative to hormone replacement therapy $[2,3]$. More recently, we demonstrated that administration of these estrogenic compounds suppressed weight gain in normal weight and obese rodents [4-6]. In particular, IP injection of various BDDA isoforms lowered body weight and total adiposity in Wistar and Sprague-Dawley rats, independently of gender or energy intake [5]. Similarly, (+)-Z-BDDA $(0.0002 \%$ food admixture) reduced weight gain and attenuated hyperglycemia and dyslipidemia in female Zucker Diabetic Fatty (ZDF) rats [4]. We also reported that (+)-Z-BDDA and related compounds exhibited significant cardiovascular protective and antioxidant properties in several studies $[4,7]$. However, the specific mechanisms involved in the antiobesity effects of these compounds remain unclear.

Our previous studies showed a marked induction of peroxisome-proliferator-activated receptor- $\alpha(\operatorname{PPAR} \alpha)$ and carnitine palmitoyltransferase-1 (CPT1) expression in liver of obese rodents administered (+)-Z-BDDA [4]. The modification of these key regulators of fatty acid metabolism is indicative of enhanced energy and substrate metabolism, which may account for the suppression of weight gain and prevention of obesity-related comorbidities. Thus, the objective of the current study was to determine how the synthetic estrogen, (+)-Z-BDDA, impacts energy expenditure and nutrient utilization in obesity. To achieve this, we evaluated 
indirect calorimetry and metabolic profile in female obese Zucker (OZR) rats provided a $0.0002 \%$ food admixture of $(+)$-Z-BDDA for 10 weeks.

\section{Material and Methods}

2.1. Animals. As previously described [4], (+)-Z-BDDA prepared from (+)-Z-BDDA-3- $\mathrm{OCH}_{3}$ was administered to female OZR $(f a / f a)$ rats (4-6 weeks of age) as $0.0002 \%$ food admixture. Animals were provided unlimited access to semipurified diet (Table 1) with or without (+)-Z-BDDA for 11 weeks $(n=8)$. All experimental protocols for animal care and use were approved by the Institutional Animal Care and Use Committee.

Body weight and energy intake were measured weekly. Nonfasted blood glucose (Glucometer Elite, Mishawaka, IN) was examined periodically to monitor progression of diabetic phenotype (data not shown). Oral glucose tolerance test (OGTT) was performed at week 10 following overnight fast (8-10 hour) [8]. At termination, rats were fasted overnight, euthanized by $\mathrm{CO}_{2}$ asphyxiation, and decapitated for collection of trunk blood in heparinized tubes. Plasma was utilized for quantitative determination of glucose, insulin, cholesterol, and triacylglyceride (TAG) concentration [4]. Tissue was also immediately extracted, weighed, and frozen for biochemical analysis.

2.2. Indirect Calorimetry. Assessment of 24-hour indirect calorimetry (Accuscan Instruments Indirect Calorimeter, Columbus, $\mathrm{OH}$ ) was performed as previously described [9]. Briefly, zirconia and infrared sensors were used to monitor oxygen $\left(\mathrm{O}_{2}\right)$ and carbon dioxide $\left(\mathrm{CO}_{2}\right)$ inside respiratory chambers. All animals were studied simultaneously using different chambers connected to the same $\mathrm{O}_{2}$ and $\mathrm{CO}_{2}$ sensors. After a 24-hour acclimation period, rats were monitored in the metabolic chambers for 24 hours with unlimited access to food and water. Gas samples were collected and analyzed every 5 minutes per animal, and the data were averaged for each hour. Output parameters included $\mathrm{VO}_{2}(\mathrm{~L} / \mathrm{d}), \mathrm{RQ}$ $\left(\mathrm{VCO}_{2} / \mathrm{VO}_{2}\right)$, and heat production $(\mathrm{kJ} / \mathrm{d})$.

2.3. Immunoblotting. Liver extracts were prepared and proteins separated by SDS page as previously described [10]. The separated proteins were transferred to a nitrocellulose membrane at $80 \mathrm{~V}$ for $90 \mathrm{~min}$. Blots were blocked overnight in $5 \%$ nonfat milk in tris-buffered saline with $0.05 \%$ Tween 20 (TBST) followed by incubation with the specified primary antibody/antibodies for $2 \mathrm{~h}$ (Santa Cruz, CA, USA). After immunoblotting, membranes were washed three times with TBST and incubated with the appropriate secondary antibody conjugated with HRP for another hour. After washing off excess secondary antibody, blots were developed with SuperSignal West Pico Chemiluminescent Substrate (Thermo Scientific, Rockford, IL, USA). X-ray film densitometry was used for quantification (BioSpectrum Imaging System, Upland, CA, USA) and signals measured with VisionWorksLS (UVP, Upland, CA, USA).
TABLE 1: Composition of experimental diets.

\begin{tabular}{lcc}
\hline & Control & $(+)-Z-$ BDDA $^{2}$ \\
& \multicolumn{2}{c}{$\mathrm{g} / \mathrm{kg}$} \\
\hline Casein $^{1}$ & 230 & 230 \\
Sucrose & 267.9 & 267.9 \\
Cornstarch & 310 & 310 \\
Soybean oil & 25 & 25 \\
Lard & 65 & 65 \\
Cellulose $^{1}$ & 50 & 50 \\
Vitamin Mix $^{1}$ & 10 & 10 \\
Mineral Mix $^{1}$ & 35 & 35 \\
Choline Bitartrate $^{1}$ & 2.5 & 2.5 \\
DL-methionine $^{1}$ & 1.6 & 1.6 \\
L-cysteine $^{1}$ & 3 & 3 \\
tert-butylhydroquinon $^{1}$ & .014 & .014 \\
(+)-Z-BDDA $^{1}$ & - & .0018 \\
\hline
\end{tabular}

${ }^{1}$ ICN Biomedicals, Irvine, CA.

${ }^{2} 0.00018 \%$ food add/mix of (+)-Z-BDDA.

TABLe 2: Metabolic parameters in female obese Zucker rats $(n=8)$ provided (+)-Z-BDDA for 11 weeks.

\begin{tabular}{lcc}
\hline Parameter & Control & $(+)-Z$-BDDA \\
\hline Final Body WT $(\mathrm{g})$ & $447 \pm 20.5$ & $390 \pm 34.0^{*}$ \\
Total WT Gain $(\mathrm{g})$ & $218 \pm 13.5$ & $190 \pm 11.9^{*}$ \\
Total Energy Intake $(\mathrm{kJ})$ & $6351 \pm 493$ & $5598 \pm 547$ \\
Liver wt (\% Body WT) & $6.40 \pm 0.15$ & $5.30 \pm 0.36^{*}$ \\
$\begin{array}{l}\text { OGTT AUC (Arbitrary } \\
\text { Units) }\end{array}$ & $632 \pm 61.5$ & $444 \pm 66.1^{*}$ \\
$\begin{array}{l}\text { Plasma Insulin (nmol/L) } \\
\text { Plasma Triglycerides } \\
\text { (mmol/L) }\end{array}$ & $1.21 \pm 0.19$ & $0.91 \pm 0.03$ \\
$\begin{array}{l}\text { Plasma Cholesterol } \\
\text { (mmol/L) }\end{array}$ & $4.24 \pm 0.51$ & $3.69 \pm 1.27$ \\
\hline
\end{tabular}

${ }^{*}$ indicates significant reduction by $(+)-Z$-BDDA treatment compared to control as determined by Student's $t$-test $(P<0.05)$.

2.4. Statistical Analysis. Data are presented as means \pm SE, compared by Student's $t$-test (SYSTAT7 10.0, SPSS Incorporated, Chicago, IL).

\section{Results}

Consistent with our previous work $[4,5],(+)-Z$-BDDA reduced body weight and total weight gain, independent of energy intake (Table 2, $P<0.05$ ). These effects corresponded with an improved metabolic phenotype in female OZR rats. In particular, liver weight and plasma total cholesterol were reduced by $18 \%$ and $56 \%$ in (+)-Z-BDDA-treated animals (Table 2, $P<0.05$ ), respectively. In addition, oral glucose tolerance was improved by approximately $30 \%$ with $(+)-Z-$ BDDA treatment. Although we did not detect improvements in plasma insulin or TAG concentration (Table 2), these data confirm that $(+)-Z$-BDDA administration partially 


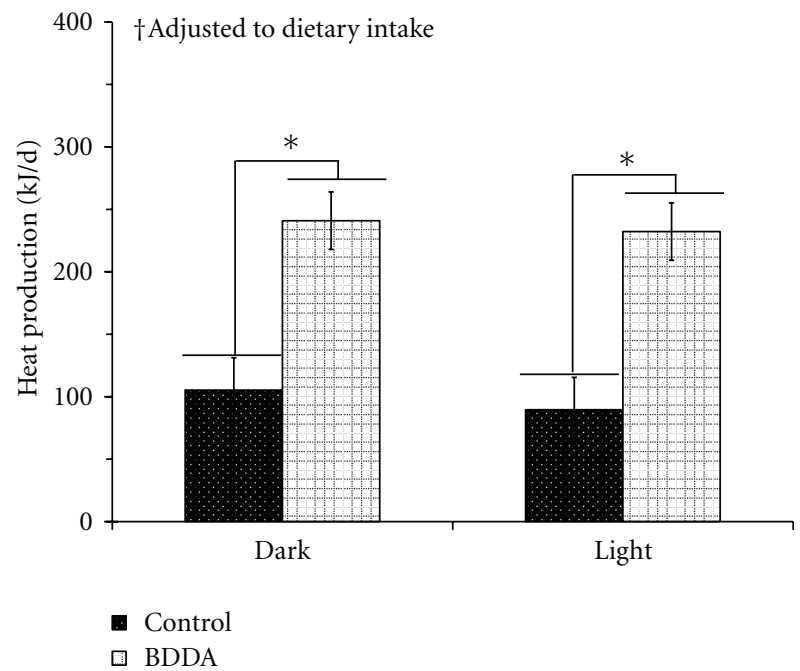

(a)

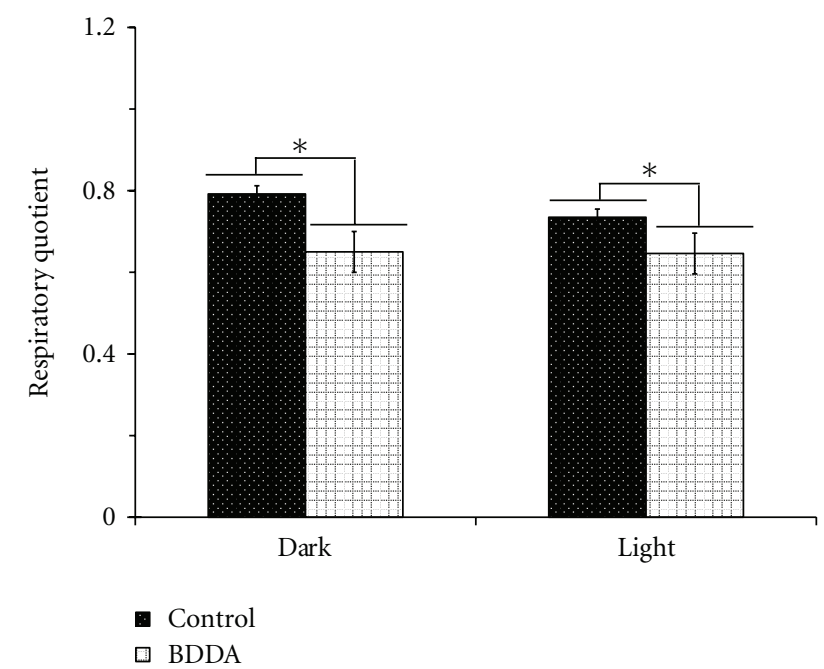

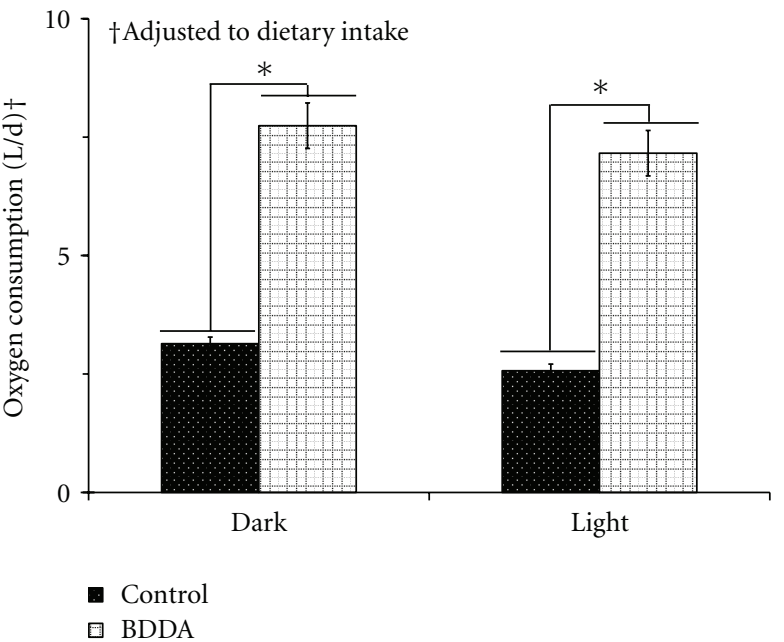

(b)

(c)

FIgure 1: (+)-Z-BDDA enhanced basal metabolism and fatty acid oxidation in female OZR rats. After a 24-hour acclimation period, rats were monitored in the metabolic chambers (Accuscan Instruments Indirect Calorimeter, Columbus, OH) for $24 \mathrm{~h}$ with unlimited access to food and water. Gas samples were collected and analyzed every 5 minutes, averaged for each hour, and presented as total for $24 \mathrm{~h}$ period. Output parameters included heat production $(\mathrm{kJ} / \mathrm{d}), \mathrm{VO}_{2}(\mathrm{~L} / \mathrm{d})$, and $\mathrm{RQ}\left(\mathrm{VCO}_{2} / \mathrm{VO}_{2}\right)$. All measures were obtained from female OZR rats after 11 weeks of $(+)$-Z-BDDA treatment $(n=8)$. Graphed values represent means \pm SE. Asterisks $\left({ }^{*}\right)$ represent significant differences among means as determined by Student's $t$-test $(P<0.05)$. (a) Heat production expressed as kilojoules per day; (b) oxygen consumption expressed as liters per day; (c) respiratory quotient (RQ).

attenuates obesity-related comorbidities through reduced weight gain.

Using 24-hour indirect calorimetry, we then examined if changes in basal metabolism and substrate utilization were responsible for the antiobesity properties of $(+)-Z$-BDDA. Here, we observed that heat production (Figure 1(a)) and oxygen consumption (Figure 1(b)) for both light and dark cycles were approximately $60 \%$ greater with (+)-Z-BDDA treatment when adjusted for dietary intake $(P<0.05)$. Furthermore, we detected markedly lower RQ values in obese rodents provided (+)-Z-BDDA (Figure $1(\mathrm{c}), P<0.05$ ).
These data indicate that (+)- $Z$-BDDA enhanced resting energy expenditure and fatty acid utilization, which likely contributed to improvements in obesity phenotype.

Our previous work [4] has shown that PPAR $\alpha$ and CPT1 expression-key regulators of fatty acid oxidation-were induced in liver by $(+)-Z$-BDDA treatment. To determine if enhanced fatty acid oxidation in liver might contribute to changes in energy expenditure, we measured activation of AMPK, an upstream regulator of fatty acid oxidation. From this analysis, we identified a nearly $40 \%$ induction of hepatic AMPK phosphorylation in (+)-Z-BDDA-treated OZR rats 


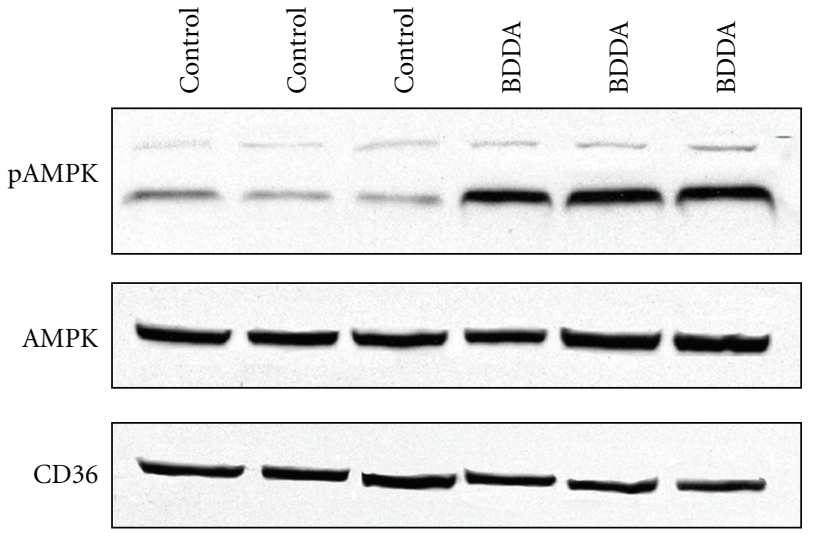

(a)

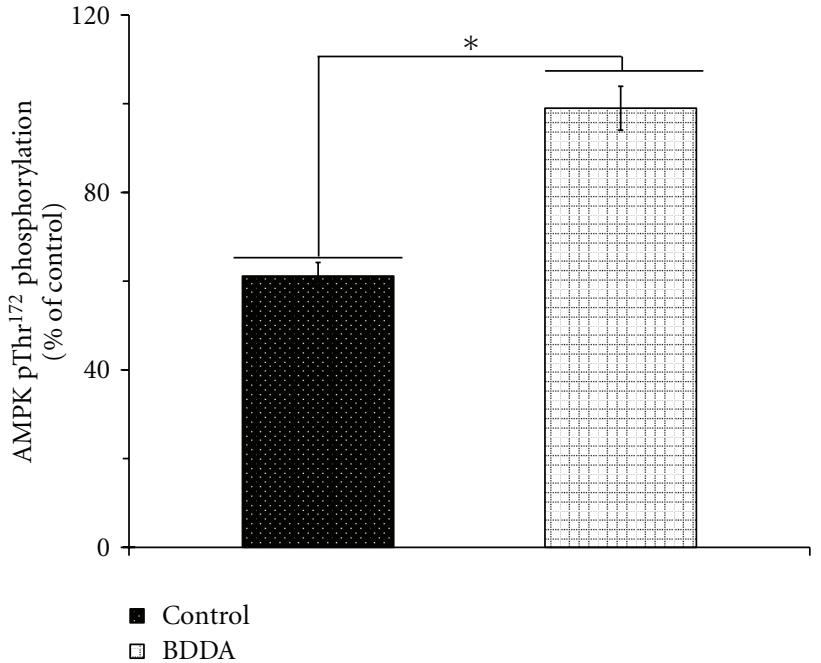

(b)

Figure 2: (+)-Z-BDDA enhanced hepatic AMPK phosphorylation in female OZR rats. Liver extracts were prepared and proteins separated by SDS page as previously described [10]. Following incubation with primary and secondary antibodies (Santa Cruz), immunoblots were developed with SuperSignal West Pico Chemiluminescent Substrate (Thermo Scientific). X-ray film densitometry was used for quantification (BioSpectrum Imaging System) and analysis (VisionWorksLS, UVP). All measures were obtained from female OZR rats after 11 weeks of $(+)$-Z-BDDA treatment $(n=4)$. (a) Hepatic AMPK phosphorylation (AMPK1/2 Thr ${ }^{172}$ ), total AMPK (AMPK $\alpha 1 / 2$ H-300), and CD36 (H$300)$ are presented as representative blots from control and (+)-Z-BDDA groups; (b) all displayed values represent percentage of control (means \pm SE). Significant differences between groups were determined by Student's $t$-test $(P<0.05)$ and indicated by asterisk $(*)$.

(Figure 2, $P<0.05)$, which occurred without changes in total AMPK (Figure 2). Thus, the antiobesity properties of $(+)-Z$ BDDA may partially reflect enhanced fatty acid in liver.

\section{Discussion}

In this study, we confirmed earlier findings [4-6] that $(+)$ $Z$-BDDA reduced weight gain, independently of energy intake. Similarly, female Zucker diabetic fatty (ZDF) rats administered (+)-Z-BDDA for six months had lower body weight, total adiposity, and enhanced physiological and cardiovascular function [4]. Here, we observed an improvement in lipid and glucose homeostasis with (+)-Z-BDDA treatment (Table 1). The augmentation of these metabolic parameters may be attributed to lower body weight and total adiposity. Additionally, (+)-Z-BDDA may attenuate the development of obesity-related pathologies independently of weight gain. For instance, estrogenic compounds have been reported to reduce inflammation in obese postmenopausal women without changes in body weight $[11,12]$. The direct impact of $(+)-Z$-BDDA and related compounds on these comorbidities should be addressed in future studies. In particular, the efficacy of (+)-Z-BDDA should be compared to natural estrogens. Our previous work with soy phytoestrogens (i.e., genistein and daidzein) has shown significant hepatoprotective properties in vivo $[13,14]$. Thus, it is possible that these compounds share a similar signaling mechanism that results in changes to energy expenditure and substrate utilization.

Obese rodents typically exhibit lower heat production and oxygen consumption compared to lean counterparts
[15]. For this study, we demonstrated that (+)-Z-BDDA treatment reduced this effect and enhanced resting energy expenditure in female OZR rats. These effects were even more apparent after normalization of heat production and oxygen consumption for dietary intake. This was an important adjustment as heat increment can be considerably influenced by differences in energy consumption [16]. In addition to changes in basal energy expenditure, we observed markedly lower RQ values with (+)-Z-BDDA treatment. Although implementation of RQ as a marker of fatty acid oxidation is not ideal [17], these values are consistent with enhanced fat oxidation. However, the impact of (+)-Z-BDDA on fatty acid utilization in obesity will need to be clarified by further analysis (i.e., non-protein $\mathrm{RQ)}$.

We previously demonstrated that (+)-Z-BDDA induced expression of PPAR $\alpha$ and CPT1 in liver of female ZDF rats [4]. Based on these findings, we measured phosphorylation of AMPK - a master regulator of fatty acid metabolism in liver. Here, we observed a marked increase in hepatic AMPK phosphorylation with (+)-Z-BDDA treatment. Although this is not a direct measure of fatty acid oxidation, the data are consistent with an induction of mitochondrial fatty oxidation. In the current study, we did not examine the potential involvement of extrahepatic tissues in regulation of these mechanisms. In particular, $17 \beta$-estradiol is reported to directly stimulate AMPK phosphorylation in primary muscle cells [18]. To address these limitations, we are currently working with primary hepatocytes and myotubes to determine how (+)-Z-BDDA modifies energy and fatty acid metabolism in these tissues. Moreover, future in vivo 
experiments need to incorporate additional measurements of AMPK induction, including the ATP to ADP ratio.

Collectively, these data-along with previous work [4, 5 - - support a potential therapeutic role for (+)-Z-BDDA in the prevention of obesity and its related comorbidities. Furthermore, we have identified that the antiobesity effects of (+)-Z-BDDA are partially mediated through enhanced resting energy expenditure and fatty acid utilization. Future work will need to address the specific cellular targets and mechanisms involved with these metabolic effects, as well as identify the potential for $(+)-Z$-BDDA and related compounds to be used as weight loss agents.

\section{Conclusions}

These findings indicate that the synthetic estrogen, (+)- $Z$ BDDA partially attenuated obesity and its related pathologies through enhancement of resting energy expenditure and fatty acid utilization. Further investigation is required to identify the specific molecular targets of these compounds as well as to determine their potential therapeutic implications in humans.

\section{Acknowledgments}

This material is based upon work supported in part by the Meyers Institute at Southern Illinois University. The content of this paper is new and solely the responsibility of the authors and does not necessarily represent the official views of the granting agencies. The authors have no conflict of interests to report. The authors would also like to extend their appreciation to PreClinOmics, Inc.

\section{References}

[1] J. E. Davis, Y. Hou, C. Y. Meyers, and W. J. Banz, "Potential clinical applications of $(-),(+)$ and $(+)-Z-$ bisdehydrodoisynolic acids in metabolic disorders," Recent Patents on Endocrine, Metabolic and Immune Drug Discovery, vol. 3, no. 3, pp. 173-178, 2009.

[2] N. G. Schneeberg, L. Perczek, J. H. Nodine, and W. H. Perloff, "Methallenestril, a new synthetic estrogen," JAMA, vol. 161, no. 11, pp. 1062-1067, 1956.

[3] L. Terenius, "Differential inhibition in vitro of 17 betaestradiol binding in the mouse uterus and vagina by optical antipodes of estrogens," Molecular Pharmacology, vol. 4, no. 4, pp. 301-310, 1968.

[4] W. J. Banz, J. Davis, J. J. Steinle et al., “(+)-Z-Bisdehydrodoisynolic acid ameliorates obesity and the metabolic syndrome in female ZDF rats," Obesity Research, vol. 13, no. 11, pp. 1915-1924, 2005.

[5] W. J. Banz, T. A. Winters, Y. Hou, S. Adler, and C. Y. Meyers, "Comparative effects of the selective estrogen receptor modulators $(-)-,(+)$-and $( \pm)$-z-bisdehydrodoisynolic acids on metabolic and reproductive parameters in male and female rats," Hormone and Metabolic Research, vol. 30, no. 12, pp. 730-736, 1998.

[6] C. Y. C. Meyers, W. J. Banz, S. R. Adler, T. A. Winters, Y. Hou, and W. B. Dandliker, Therapeutic Applications of Estrogenic
Carboxylic Acids, Southern Illinois University, Carterville, Ill, USA, 2004.

[7] T. Wilson, H. March, W. J. Banz et al., "Antioxidant effects of phyto-and synthetic-estrogens on cupric ion-induced oxidation of human low-density lipoproteins in vitro," Life Sciences, vol. 70, no. 19, pp. 2287-2297, 2002.

[8] A. D. Strader, T. R. Clausen, S. Z. Goodin, and D. Wendt, "Ileal interposition improves glucose tolerance in low dose streptozotocin-treated diabetic and euglycemic rats," Obesity Surgery, vol. 19, no. 1, pp. 96-104, 2009.

[9] R. Westbrook, M. S. Bonkowski, A. D. Strader, and A. Bartke, "Alterations in oxygen consumption, respiratory quotient, and heat production in long-lived GHRKO and Ames dwarf mice, and short-lived bGH transgenic mice," Journals of Gerontology-Series A Biological Sciences and Medical Sciences, vol. 64, no. 4, pp. 443-451, 2009.

[10] Y. Zheng, W. Zhang, E. Pendleton et al., "Improved insulin sensitivity by calorie restriction is associated with reduction of ERK and p70S6K activities in the liver of obese Zucker rats," Journal of Endocrinology, vol. 203, no. 3, pp. 337-347, 2009.

[11] L. Azadbakht, M. Kimiagar, Y. Mehrabi, A. Esmaillzadeh, F. B. $\mathrm{Hu}$, and W. C. Willett, "Soy consumption, markers of inflammation, and endothelial function: a cross-over study in postmenopausal women with the metabolic syndrome," Diabetes Care, vol. 30, no. 4, pp. 967-973, 2007.

[12] Y. Huang, S. Cao, M. Nagamani, K. E. Anderson, J. J. Grady, and L. J. W. Lu, "Decreased circulating levels of tumor necrosis factor- $\alpha$ in postmenopausal women during consumption of soy-containing isoflavones," The Journal of Clinical Endocrinology and Metabolism, vol. 90, no. 7, pp. 3956-3962, 2005.

[13] J. Cain, W. J. Banz, D. Butteiger, and J. E. Davis, "Soy protein isolate modified metabolic phenotype and hepatic Wnt signaling in obese Zucker rats," Hormone and Metabolic Research, vol. 43, no. 11, pp. 774-781, 2011.

[14] O. Mezei, W. J. Banz, R. W. Steger, M. R. Peluso, T. A. Winters, and N. Shay, "Soy isoflavones exert antidiabetic and hypolipidemic effects through the PPAR pathways in obese Zucker rats and murine RAW 264.7 cells," Journal of Nutrition, vol. 133, no. 5, pp. 1238-1243, 2003.

[15] J. J. Ramsey, D. E. Johnson, K. L. Hossner, and K. A. Johnson, "Metabolic rate, organ mass, and mitochondrial proton leak variations in lean and obese rats," Comparative Biochemistry and Physiology $-B$ Biochemistry and Molecular Biology, vol. 113, no. 3, pp. 461-466, 1996.

[16] C. K. Martin, L. K. Heilbronn, L. De Jonge et al., "Effect of calorie restriction on resting metabolic rate and spontaneous physical activity," Obesity, vol. 15, no. 12, pp. 2964-2973, 2007.

[17] E. Jéquier, K. Acheson, and Y. Schutz, "Assessment of energy expenditure and fuel utilization in man," Annual Review of Nutrition, vol. 7, pp. 187-208, 1987.

[18] N. H. Rogers, C. A. Witczak, M. F. Hirshman, L. J. Goodyear, and A. S. Greenberg, "Estradiol stimulates Akt, AMP-activated protein kinase (AMPK) and TBC1D1/4, but not glucose uptake in rat soleus," Biochemical and Biophysical Research Communications, vol. 382, no. 4, pp. 646-650, 2009. 


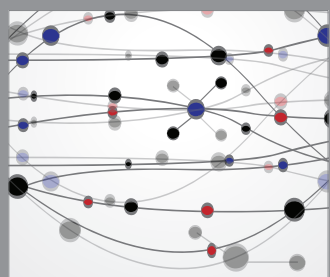

The Scientific World Journal
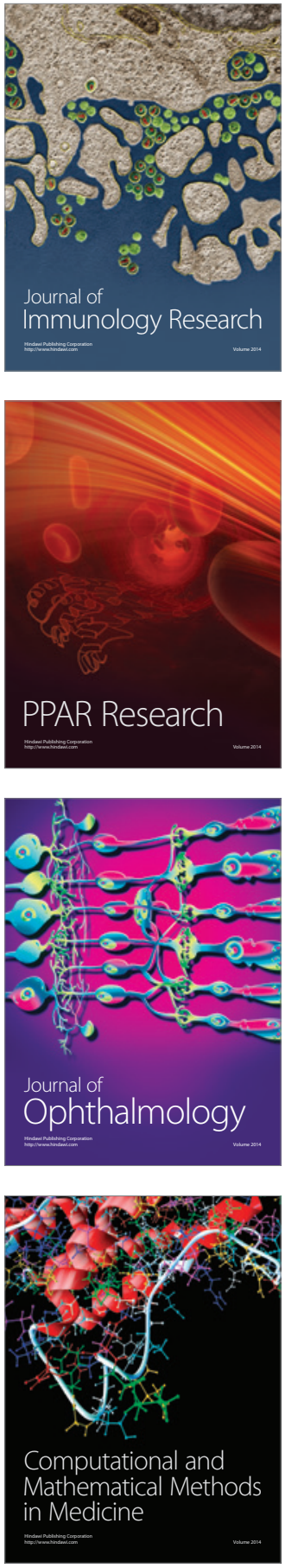

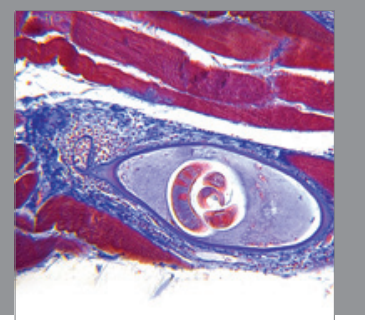

Gastroenterology

Research and Practice
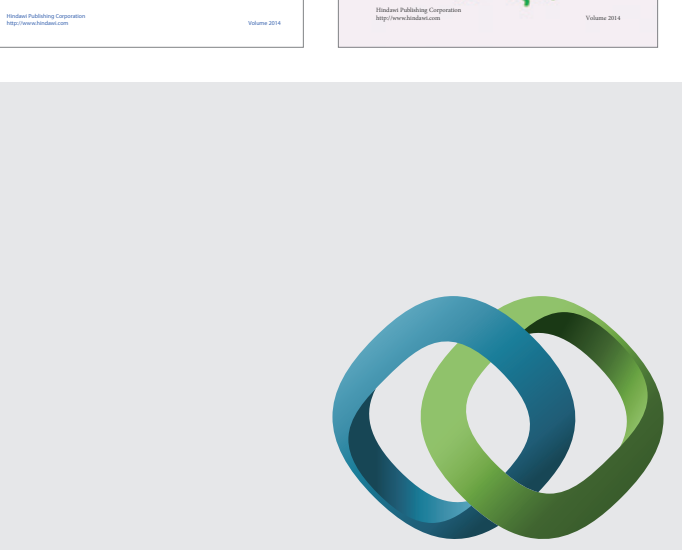

\section{Hindawi}

Submit your manuscripts at

http://www.hindawi.com
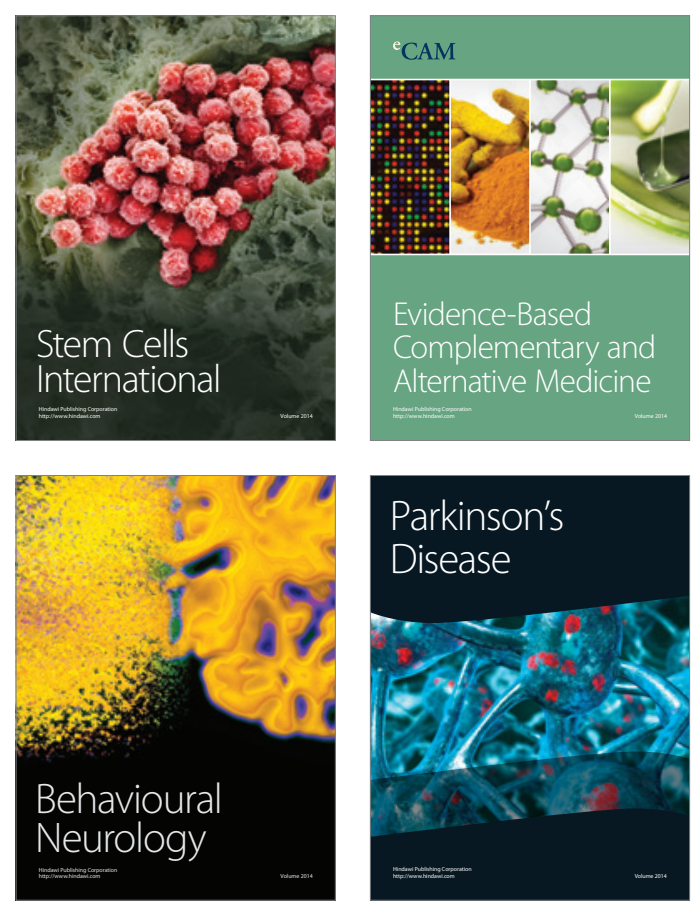

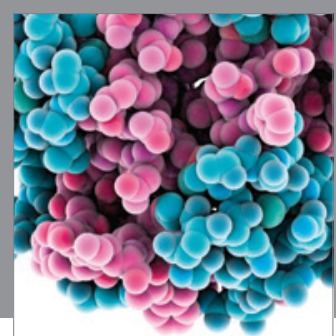

Journal of
Diabetes Research

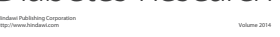

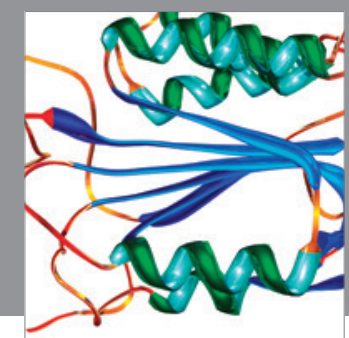

Disease Markers
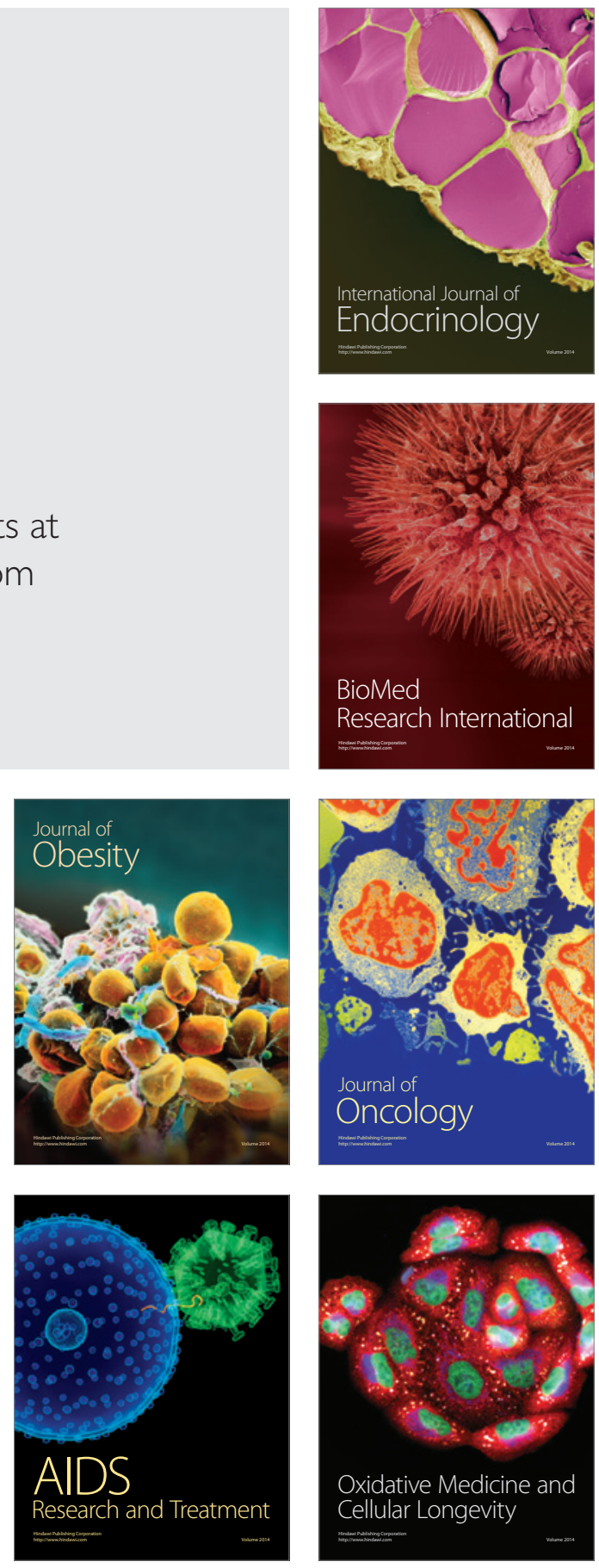